\title{
POTENTIAL ISOMORPHISM OF ELEMENTARY SUBSTRUCTURES OF A STRICTLY STABLE HOMOGENEOUS MODEL
}

\author{
SY-DAVID FRIEDMAN, TAPANI HYTTINEN, AND AGATHA C. WALCZAK-TYPKE
}

\begin{abstract}
The results herein form part of a larger project to characterize the classification properties of the class of submodels of a homogeneous stable diagram in terms of the solvability (in the sense of [Fri03]) of the potential isomorphism problem for this class of submodels.

We restrict ourselves to locally saturated submodels of the monster model $\boldsymbol{m}$ of some power $\pi$. We assume that in Gödel's constructible universe $\mathbb{L}, \pi$ is a regular cardinal at least the successor of the first cardinal in which $\mathbf{m}$ is stable.

We show that the collection of pairs of submodels in $\mathbb{L}$ as above which are potentially isomorphic with respect to certain cardinal-preserving extensions of $\mathbb{L}$ is equiconstructible with $0^{\#}$. As $0^{\#}$ is highly "transcendental" over $\mathbb{L}$, this provides a very strong statement to the effect that potential isomorphism for this class of models not only fails to be set-theoretically absolute, but is of high (indeed of the highest possible) complexity.

The proof uses a novel method that does away with the need for a linear order on the skeleton.
\end{abstract}

\section{CONTENTS}

1. Introduction

2. Preliminaries

2.1. Notation

2.2. Set theory

2.2.1. Relative constructibility

2.3. Homogeneous model theory

2.3.1. Introduction and motivation for homogeneous model theory

2.3.2. Types and homogeneous monsters

2.3.3. Indiscernibles and strong splitting independence

2.3.4. Primary model constructions

3. The Strictly Stable Case

3.1. Defining the trees and other orderings

The first author wishes to thank the Fonds zur Förderung der wissenschaftlichen Forschung (FWF) for its support through grant P19898-N18 and the John Templeton Foundation for its support through the CRM Infinity Project, Grant \#13152.

The second and third authors gratefully acknowledge the hospitality of the Institut MittagLeffler, Djursholm, Sweden during Fall 2009.

The work of the second and third authors is partially supported by the Academy of Finland, grant number 1123110 .

This paper will appear in the September 2011 issue of the Journal of Symbolic Logic. 
3.1.1. The first generation of trees

3.1.2. The second generation of trees $\quad 9$

3.1.3. The third generation: a quasi-order $r$

3.2. Building the models: putting fat on the trees 11

3.2.1. Cardinal assumptions 11

3.2.2. An initial $\omega$-sequence of models 11

3.2.3. The construction $\quad 12$

3.3. Non-isomorphism when symmetric difference of $S$-invariants is stationary 14

3.4. Isomorphism of the models when $S$ is killed 16

3.5. Constructibility with respect to $0^{\#} \quad 20$

References $\quad 20$

\section{INTRODUCTION}

The results we give here are part of a larger project to prove strong nonstructure results for non-elementary classes. The original impetus comes from work to generalize the results of [FHR03] to the Homogeneous Model Theory context. The main theorem of that earlier work was:

Theorem ([FHR03]). Assume $0^{\#}$ exists, and let $T$ be a constructible first-order theory which is countable in Gödel's constructible universe $\mathbb{L}$. Then the following are equivalent:

(1) The collection

(1) $\left\{\langle\mathscr{A}, \mathscr{B}\rangle \in \mathbb{L}: \mathscr{A} \models T, \mathscr{B} \models T, \mathscr{A}, \mathscr{B}\right.$ have universe $\left(\aleph_{2}\right)^{\mathbb{L}}$, and are isomorphic in an extension of $\mathbb{L}$ with the same

cardinals and reals as $\mathbb{L}\}$

is constructible.

(2) The theory $T$ is superstable with NOTOP and NDOP.

This result was proved using strong non-structure theorems, following the cases found in the Main Gap Theorem [She90].

We chose the Homogeneous Model Theory context to extend this result because of its well developed Main Gap Theorem [HS01]. Much of the difficulty lies in finding strong non-structure theorems in the Homogeneous Model Theory (HMT) context. While one can prove strong non-structure theorems in nonelementary contexts (e.g. Abstract Elementary Classes, or as in [GS86]) having the order property in exactly the same way as was done for unstable first order theories, strong non-structure theorems have not been proved for almost any other non-elementary classes. This is because the only first-order strong nonstructure theorem that can be generalized in a straight-forward manner is the one stemming from the order property.

In this paper, we prove a strong non-structure theorem for the strictly stable (stable but not superstable) case in HMT. In the first-order context, non-structure 
theorems for the strictly stable case are proved by first finding tree indiscernibles, and then using them as skeleta in Ehrenfeucht-Mostowski model constructions. In the HMT context, a major problem arises in simply generalizing the approach used in the first-order context: without large cardinals one cannot find treeindiscernibles. Thus if one wants to carry out the constructions in $\mathbb{L}$, as we do in this paper, currently known methods do not allow for the use of EhrenfeuchtMostowski model constructions. Alternatively, if one were willing to assume large cardinals, then the ideas of [Fri03] would have to be generalized from $\mathbb{L}$ to a larger core model, raising significant new set-theoretic challenges.

We hope that our exposition will be accessible both to model theorists and to set theorists. Those seeking definitions of set-theoretic concepts should consult, for example, [Jec03]. On the other side, [Hyt97b] or a similar introduction to methods in classification theory may help with the model-theoretic concepts. However, full comprehension of this paper requires knowledge of [HS98].

\section{Preliminaries}

2.1. Notation. Gödel's constructible universe will be denoted as $\mathbb{L}$. To differentiate, similarity types (languages) will be denoted with the calligraphic $\mathcal{L}$.

\subsection{Set theory.}

2.2.1. Relative constructibility. This paper is concerned with examining the solvability (in the sense of [Fri03]) of certain problems in the classification of structures that are not-first order axiomatizable. Our intuition is that if the collection of constructible objects that satisfy a particular condition is constructible (i.e. in $\mathbb{L})$, then we say that the condition's problem is solvable. On the other hand, if the collection is not in $\mathbb{L}$, then we say that the condition's problem is unsolvable.

We will demonstrate the unsolvability of a problem by reducing to it sets that are known to be non-constructible - indeed, to sets that are equiconstructible with $0^{\#}$.

First, some notation:

Definition 2.1. We have the following notion of reduction:

Suppose that $\left\langle X_{0}, X_{1}\right\rangle,\left\langle Y_{0}, Y_{1}\right\rangle$ are pairs of disjoint subsets of the constructible universe $\mathbb{L}$. That is, that they are disjoint collections of constructible sets. Note that the pairs $\left\langle X_{0}, X_{1}\right\rangle$ and $\left\langle Y_{0}, Y_{1}\right\rangle$ need not be constructible themselves. We write

$$
\left\langle X_{0}, X_{1}\right\rangle \stackrel{\mathbb{L}}{\rightarrow}\left\langle Y_{0}, Y_{1}\right\rangle
$$

if there exists a constructible function $g \in L$ such that

$$
x \in X_{0} \Rightarrow g(x) \in Y_{0} \text { and } x \in X_{1} \Rightarrow g(x) \in Y_{1} .
$$

We write $X_{0}$ instead of $\left\langle X_{0}, X_{1}\right\rangle$ in the case that $X_{0}$ is the complement of $X_{1}$ within some constructible set. We employ the analogous convention for the Ys. 
The idea behind this notion of reduction is that if $\left\langle X_{0}, X_{1}\right\rangle$ is non-constructible, $X_{0} \cup X_{1}$ is constructible, and $\left\langle X_{0}, X_{1}\right\rangle \stackrel{\mathbb{L}}{\rightarrow}\left\langle Y_{0}, Y_{1}\right\rangle$, then $\left\langle Y_{0}, Y_{1}\right\rangle$ is also non-constructible.

\section{Definition 2.2.}

(1) A cardinal preserving extension ("Cap-extension") of $\mathbb{L}$ is a transitive model satisfying the Axiom of Choice containing all the ordinals, and which is contained in a set-generic extension of $V$ and has the same cardinals as $\mathbb{L}$.

(2) A cardinal- and real-preserving extension ("CaRp-extension") of $\mathbb{L}$ is a transitive model satisfying the Axiom of Choice containing all the ordinals, and which is contained in a set-generic extension of $V$ and has the same cardinals and real numbers as $\mathbb{L}$.

(3) For $\nu$ a cardinal, a cardinal- and $\mathscr{P}(\nu)$-preserving extension ("Ca $\mathscr{P}(\nu)$ extension") is defined analogously.

We also remind the reader of the following highly non-constructible object:

Definition 2.3. If there exists a non-trivial elementary embedding of the constructible universe $\mathbb{L}$ into itself, then there is a closed unbounded proper class of ordinals that are indiscernible for the structure $(\mathbb{L}, \in)$. Then, we can define $0^{\#}$ ("zero-sharp") to be the real number that codes in the canonical way the Gödel numbers of the formulas that are true about the indiscernibles in $\mathbb{L}$.

The existence of $0^{\#}$ is independent of the axioms of set theory, ZFC. If ZFC is consistent, then so is ZFC with the assumption that $0^{\#}$ does not exist. It is commonly assumed that ZFC is consistent with the assumption that $0^{\#}$ does exist.

We assume throughout that $0^{\#}$ exists.

The real number $0^{\#}$ is highly non-constructible object. Our intuition will be to show that a class of models is non-constructible by reducing $0^{\#}$ to it, in the sense above. In particular, we will use the following theorem. We denote by $S_{\omega}^{\nu}$ the stationary set consisting of ordinals in $\nu$ of cofinality $\omega$.

Theorem 2.4 ([Fri03]). Denote by $\mathcal{S}(\kappa)$ [resp. $\left.\mathcal{S}_{r}(\kappa)\right]$ the collection of sets $S \in \mathbb{L}$ such that $S \subseteq\left(S_{\omega}^{\nu}\right)^{\mathbb{L}}$ is stationary in $\mathbb{L}$ and in a cardinal- [and real-] preserving extension, $\nu \backslash S$ contains a club.

Then, if $\kappa$ is an uncountable regular cardinal in $\mathbb{L}$ and $\left(\kappa^{+}=\nu\right)^{\mathbb{L}}$, then

$$
0^{\#} \stackrel{\mathbb{L}}{\rightarrow} \mathcal{S}(\kappa)
$$

and

$$
0^{\#} \stackrel{\mathbb{L}}{\rightarrow} \mathcal{S}_{r}(\kappa)
$$




\subsection{Homogeneous model theory.}

2.3.1. Introduction and motivation for homogeneous model theory. Homogeneous Model Theory (HMT), introduced in [She70] as "finite diagrams stable in power", is an approach to the model-theoretic classification of classes of nonelementary structures (i.e. structures not axiomatizable using a first-order theory). The motivation behind the development of this approach, as explained in [HS01, GL02], was the aim to classify the class of models of an $\mathcal{L}_{\gamma^{+} \omega}$ sentence $\psi$, with $\preccurlyeq \mathcal{L}_{\gamma^{+}}$as the substructure relation. We wish this class of models to be "well behaved" and so add the requirement that the class satisfies the amalgamation property. It was proved in [She70] that it is equivalent to consider the class of elementary submodels of a homogeneous monster model $\mathrm{m}$.

Thus, in practice the contrast to elementary (first-order) model theory where one assumes that all considerations take place within a large saturated monster model, is that we take away the assumption that the monster is saturated, and instead only insist that it be homogeneous. However, in the HMT context, a major difficulty arises because the compactness theorem fails. In return for this concession, we do gain a widening of the possible structures under consideration as opposed to elementary model theory. For example, the class of existentially closed models of an inductive theory can be studied within the framework of homogeneous model theory. In fact, for some $\gamma$ big enough the class of submodels of a homogeneous model can be axiomatized in some theory $T^{*} \subset \mathcal{L}_{\gamma^{+} \omega}$. (Specifically, where $\gamma \geqslant|D(\operatorname{Th}(\mathbf{m})) \backslash D|$, where $D$ is the finite diagram. For more specifics, see [She70, GL02].)

2.3.2. Types and homogeneous monsters. We assume we work within very large homogeneous model which can serve as a monster model. We will then be interested in the class of elementary submodels of this monster.

We work with m-consistent types:

Definition 2.5 ([HS01]). Let $A \subseteq \mathbf{m}_{\text {, }}$, and let $p$ be a (first-order) type over $A$. We say that $p$ is $\boldsymbol{m}$-consistent if it is realized in $\mathbf{m}$.

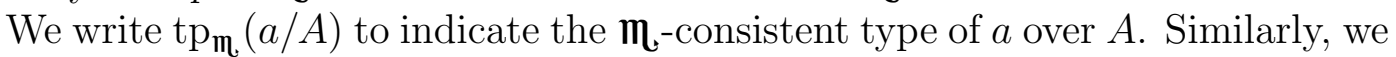
take $\mathrm{S}_{\mathfrak{m}}^{m}(A)=\left\{\operatorname{tp}_{\mathfrak{m}}(a / A): a \in \mathbf{m}_{\mathbf{l}}\right.$, length $\left.(a)=m\right\}$, and $\mathrm{S}_{\mathfrak{m}}(A)=\bigcup_{m<\omega} \mathrm{S}_{\mathfrak{m}}^{m}(A)$.

Definition 2.6. A homogeneous monster model $m_{\text {o }}$ is said to be stable in $\lambda$ if for every $B \subset \operatorname{dom}(\mathbf{m})$ of cardinality at most $\lambda$, and for every $n<\omega$, we have $\left|\mathrm{S}_{\mathrm{m}}^{n}(B)\right| \leqslant \lambda$.

The monster model $\boldsymbol{m}_{\mathbf{m}}$ is stable if it is stable in some $\lambda$.

The monster model $\mathbf{m}$ is unstable if it is not stable.

We denote by $\lambda(\mathbf{m})$ the least $\lambda$ in which $\mathbf{m}$ is stable, if it exists [HS00]. Denote by $\lambda_{r}(\mathbf{m})$ the first regular cardinal $\geqslant \lambda(\mathbf{m})$.

2.3.3. Indiscernibles and strong splitting independence. A standard notion from model theory follows. We include this definition to make the terminology clear, 
as the set-theoretic usage is sometimes at odds with accepted usage among model theorists.

Definition 2.7. An (indexed) set of tuples $\left\{\bar{a}_{i}: i<\alpha\right\}$ is called an $n$-indiscernible sequence over $A$, for $n<\omega$, if

$$
\operatorname{tp}\left(\bar{a}_{0}, \ldots, \bar{a}_{n-1} / A\right)=\operatorname{tp}\left(\bar{a}_{i_{0}}, \ldots, \bar{a}_{i_{n-1}} / A\right),
$$

for every $i_{0}<\cdots<i_{n-1}<\alpha$. The set of tuples $\left\{\bar{a}_{i}: i<\alpha\right\}$ is an indiscernible sequence over $A$ if it is an $n$-indiscernible sequence over $A$ for every $n<\omega$. It is said to be an indiscernible set if the ordering induced by the indices does not matter.

Definition 2.8 ([She90] III, p. 85, Def. 1.2). A type $p \in \mathrm{S}^{n}(A)$ splits strongly over $B \subseteq A$ if there exists $\left\{\bar{a}_{i}: i<\omega\right\}$ an indiscernible sequence over $B$ and a formula $\phi$ such that $\phi\left(\bar{x}, \bar{a}_{0}\right), \neg \phi\left(\bar{x}, \bar{a}_{1}\right) \in p$.

The following definitions are very similar to the definitions of independence and $\kappa(T)$ in the first-order context. However, here we use strong splitting instead of forking in the definitions. In the first order context, the definitions using forking and the definition as stated here are equivalent. In the HMT context, forking is ill-defined, so we take the strong splitting definition. Consequently, we lose some nice properties, among them transitivity of the independence relation.

Definition 2.9 ([HSO0], p. 2). We define $\kappa\left(\mathbf{m}_{\mathbf{c}}\right)$ to be the least infinite cardinal such that there are no $a, b_{i}$, and $c_{i}, i<\kappa(\mathbf{m} \mathbf{)}$, such that

(i) for all $i<\kappa(\mathbf{m})$, there is an infinite indiscernible set $I_{i}$ over $\bigcup_{j<i}\left(b_{j} \cup c_{j}\right)$ such that $b_{i}, c_{i} \in I_{i}$,

(ii) for all $i<\kappa(\mathbf{m})$, there is $\phi_{i}(x, y)$ such that $\models \phi_{i}\left(a, b_{i}\right) \wedge \neg \phi_{i}\left(a, c_{i}\right)$.

Note that $\kappa(\mathbf{m}) \leqslant \lambda(\mathbf{m} \mathbf{})$ by Corollary 1.3 of [HS00].

Definition 2.10 ([HS00], p. 17, remarks before Lemma 5.1). We say that a monster model is superstable if $\kappa\left(\mathbf{m}_{\mathbf{c}}\right)=\aleph_{0}$. We will call a monster model strictly stable if it is stable, but not superstable.

Now we can define the notion of independence that we use in the HMT context.

Definition 2.11 ([HS00], Def. 3.1(i)). We write $a \downarrow_{A} B$ if there is $C \subseteq A$, $|C|<\kappa\left(\mathbf{m}_{\mathbf{l}}\right)$, such that for all $D \supseteq A \cup B$ there is $b$ which satisfies $\operatorname{tp}_{\mathbf{m}}(b / A \cup B)=$ $\operatorname{tp}_{\mathrm{m}}(a / A \cup B)$ and $\operatorname{tp}_{\mathrm{m}}(b / D)$ does not split strongly over $C$. We write $C \downarrow_{A} B$ if for all $a \in C, a \downarrow_{A} B$.

2.3.4. Primary model constructions. Most of the following definitions are given only in very general terms that allow one to apply the notions to a very wide range of contexts. We give here these definitions specifically in the way we need them in our context.

Definition 2.12. For the following, $\nu$ is a cardinal. 
- We say that $\operatorname{tp}_{\mathrm{m}}(a / A)$ is $\mathbf{F}_{\nu}^{\mathrm{m}}$-isolated over $B$ if there is $B \subseteq A,|B|<\nu$, such that for all $b, \operatorname{tp}_{\mathbf{m}}(b / B)=\operatorname{tp}_{\mathbf{m}}(a / B)$ implies $\operatorname{tp}_{\mathbf{m}}(b / A)=\operatorname{tp}_{\mathbf{m}}(a / A)$. ([HS00], Def. 5.2).

- We say that an (elementary sub-)model $\mathscr{A}$ (of $\mathbf{m}_{\mathbf{c}}$ ) is $\mathbf{F}_{\nu}^{\mathrm{m}}$-saturated if for all $A \subseteq \mathscr{A},|A|<\nu$, and $a$, there is $b \in \mathscr{A}$ such that $\operatorname{tp}_{\mathrm{m}}(b / A)=$ $\operatorname{tp}_{\mathfrak{m}}(a / A)$. ([HSO0], Def. 1.8(i)).

- An $\mathbf{F}_{\nu}^{\mathrm{m}}$-construction is a triple

$$
\mathscr{A}=\left\langle A,\left\{\bar{a}_{i}: i<\alpha\right\},\left\langle B_{i}: i<\alpha\right\rangle\right\rangle,
$$

such that $\operatorname{tp}_{\mathfrak{m}}\left(\overline{a_{i}} / \bigcup\left\{\overline{a_{j}}: j<i\right\} \cup A\right)$ is $\mathbf{F}_{\nu}^{\mathrm{m}}$-isolated over $B_{i}$. ([She90] IV, p. 155, Def. 1.2(1)).

- We say that $C_{0}$ is $\mathbf{F}_{\nu}^{\mathrm{m}}$-constructible over $A_{0}$ if there is some $\mathbf{F}_{\nu}^{\mathrm{m}}$-construction

$$
\mathscr{A}=\left\langle A_{0},\left\{\overline{a_{i}}: i<\alpha\right\},\left\langle B_{i}: i<\alpha\right\rangle\right\rangle
$$

such that

$$
C_{0}=\bigcup\left\{\bar{a}_{i}: i<\alpha\right\} \cup A_{0} .
$$

([She90] IV, p. 156, Def. 1.3).

- If $C$ is $\mathbf{F}_{\nu}^{\mathrm{m}}$-constructible over $A$ and $C$ is $\mathbf{F}_{\nu}^{\mathrm{m}}$-saturated then we say that $C$ is $\mathbf{F}_{\nu}^{\mathrm{m}}$-primary over $A$. ([She90] IV, p. 156, Def. 1.4(1)).

- We say that $C$ is $\mathbf{F}_{\nu}^{\mathrm{m}}$-primitive over $A$ if $A \subseteq C$, and for every $\mathbf{F}_{\nu}^{\mathrm{m}_{-}}$ saturated $C^{\prime}$ such that $A \subseteq C^{\prime}$, there is an elementary mapping $f$ from $C$ into $C^{\prime}$, where $f \Gamma_{A}$ is the identity. ([She90] IV, p. 156, Def. 1.4(2)).

- We say that $C$ is $\mathbf{F}_{\nu}^{\mathrm{m}}$-prime over $A$ if it is $\mathbf{F}_{\nu}^{\mathrm{m}}$-primitive over $A$ and $\mathbf{F}_{\nu}^{\mathrm{m}}$-saturated.

- We say $A$ is $\mathbf{F}_{\nu}^{\mathrm{m}}$-atomic over $B$ if $B \subseteq A$ and for every $\bar{a} \in A$, tp $\operatorname{ta}_{\mathrm{m}}(\bar{a} / B)$ is $\mathbf{F}_{\nu}^{\mathrm{m}}$-isolated. ([She90] IV, p. 157, Def. 1.5).

Remark 2.13. On the surface, the isolation notion $\mathbf{F}_{\nu}^{\mathrm{m}}$ above is quite similar to the isolation notion $\mathbf{F}_{\nu}^{p}$ of IV Definition 2.6 (p. 168) of [She90], an isolation notion that does not satisfy certain axioms key in constructions.

However, as was noted in the last paragraph of the introduction to [HS01], under the assumption that $\mathbf{m}$ is stable, one can easily show that the isolation notion $\mathbf{F}_{\nu}^{\mathrm{m}}$, for $\nu \geqslant \lambda_{r}\left(\mathbf{m}_{\mathbf{l}}\right)$ has properties very similar to the much better-behaved notion $\mathbf{F}_{\nu}^{s}$, a definition of which can be found in [She90] IV Definitions 2.1.1.ii and 2.1.2.

In our considerations, we will use (mostly) without comment properties of the isolation $\mathbf{F}_{\nu}^{\mathbf{m}_{\mathrm{L}}}, \nu \geqslant \lambda_{r}\left(\mathbf{m}_{\mathbf{}}\right)$ which are proved in [HS00].

Definition 2.14 ([HS01] Def. 0.1). A model $\mathscr{A}$ is said to be locally $\mathbf{F}_{\nu}^{\mathrm{m}}$-saturated if for all finite sets $A \subset \mathscr{A}$ there is a $\mathbf{F}_{\nu}^{\mathrm{m}}$-saturated model $\mathscr{B}$ such that $A \subset \mathscr{B} \subset \mathscr{A}$. 


\section{The Strictly Stable Case}

Theorem 3.1. Assume $0^{\#}$ exists.

Suppose $\mathcal{L} \in \mathbb{L}$ is a signature such that $(|\mathcal{L}| \leqslant \omega)^{\mathbb{L}}$. Let $\mathbf{m} \in \mathbb{L}$ be a strictly stable (stable, but not superstable) homogeneous monster model in similarity type $\mathcal{L}$ such that $\left(\left|\mathbf{m}_{\mathbf{l}}\right|=\mu\right)^{\mathbb{L}}$, for $\mu$ sufficiently large.

Let $\pi$ be such that $\pi=\operatorname{cf}(\pi)>\lambda_{r}(\mathbf{m})$.

Let ${ }^{\operatorname{Ca} \mathscr{P}\left(\lambda_{r}\right)}$ PIP ${ }_{\pi}^{\mathrm{m}}$ be the collection of pairs $(\mathscr{A}, \mathscr{B}) \in \mathbb{L}$ of locally $\mathbf{F}_{\left.\lambda_{r}(\mathrm{~m})\right)^{-}}^{\mathrm{m}_{\mathbf{l}}}$ saturated elementary substructures of $\mathrm{m}$. with universe $\pi$ such that there is a cardinal- and $\mathscr{P}\left(\lambda_{r}(\mathbf{m})\right)$-preserving extension of $\mathbb{L}$ in which $\mathscr{A} \cong \mathscr{B}$. (Here "PIP" stands for "potentially isomorphic pairs".)

Then, ${ }^{C a \mathscr{P}\left(\lambda_{r}\right)}$ PIP ${ }_{\pi}^{\mathrm{m}}$ is equiconstructible with $0^{\#}$.

We will show that for each stationary set $S \subseteq S_{\omega}^{\pi}$, one can find two models $\mathscr{A}, \mathscr{B} \in \mathbb{L}$ of size $\pi$ such that in any Cap-extension of $\mathbb{L}, \mathscr{A} \cong \mathscr{B}$ iff $\pi \backslash S$ contains a club set. We do this by constructing two trees of small height $J_{0}, J_{1}$, differing from one another only in that one codes $S$ while the other does not. We will then perform a primary model constructions along these trees. We show then that these models are not isomorphic in the ground model, but become isomorphic in an suitable extension only if $S$ is no longer stationary in that extension.

3.1. Defining the trees and other orderings. We define two trees $I_{0}$ and $I_{1}$, which will be used to define two trees $J_{0}$ and $J_{1}$. From $J_{0}$ and $J_{1}$ we will construct models $\mathscr{A}_{J_{0}}$ and $\mathscr{A}_{J_{1}}$, respectively, which are potentially isomorphic but not isomorphic. The trees $I_{i}, J_{i}, i=0,1$ all belong to a certain general family of trees $K_{t r}^{\omega}$, defined below. Note that the trees we define here are precisely the trees that were used for the Ehrenfeucht-Mostowski constructions in the first order strictly stable case as analyzed in [FHR03] and papers cited there.

Unlike the first-order context, without large cardinal assumptions non-structure results for strictly stable theories have only been shown for weakly $\mathbf{F}_{\lambda_{r}\left(\mathbf{m}_{\mathrm{e}}\right)^{-}}^{\mathrm{m}_{\mathrm{L}}}$ saturated models, and not in general [HS98, HS01, Hyt97a]. Ehrenfeucht-Mostowski constructions yield models that are insufficiently saturated to be able to use the existing non-structure results. We will thus instead use the technique of primary model constructions, which yield more saturated models. In addition, we cannot use Ehrehnfeucht-Mostowski constructions in this case because we would need to find tree indiscernibles in the model, and to do so we would need large cardinals that are not available to us in $\mathbb{L}$. Because we need this different technique, we need to further define $K_{i}=\mathscr{P}^{<\omega}\left(J_{i}\right)$, the set of all finite subsets of $J_{i}, i=0,1$, as well as an ordering on the $K_{i}$. We will then carry out primary model constructions using sets indexed by the $K_{i}$.

We define first a general family of trees:

Definition 3.2. Let $\theta$ be a linear order, and let $\leqslant^{\leqslant} \theta$ be the set of all suborders of $\theta$ of length at most $\omega$. We let $K_{t r}^{\omega}(\theta)$ be the class of models that are isomorphic 
to a model of the form

$$
\mathcal{I}=\left(I, \lessdot, \mathrm{DOM}_{\alpha},<_{l e x}, \operatorname{MaxInSg}\right)_{\alpha \leqslant \omega},
$$

where

(1) $I \subseteq \leqslant \omega \theta$ and is closed under initial segments;

(2) $\lessdot$ is the initial segment relation;

(3) $\operatorname{DOM}_{\alpha}=\{\eta \in I: \operatorname{dom} \eta=\alpha\}$;

(4) $<_{\text {lex }}$ denotes the lexicographic ordering on $I$;

(5) $\operatorname{Max} \operatorname{InSg}(\zeta, \eta)$ is the maximal common initial segment of $\zeta$ and $\eta$.

Trees in the class $K_{t r}^{\omega}(\theta)$ are called ordered trees in the literature. We define

$$
K_{t r}^{\omega}=\bigcup\left\{K_{t r}^{\omega}(\theta): \theta \text { is a linear order }\right\} .
$$

3.1.1. The first generation of trees. We fix some notation.

- Let $\left(\lambda=\lambda_{r}(\mathbf{m})\right)^{\mathbb{L}}$. Because we have assumed that $\mathbf{m}$ is strictly stable, $\lambda \geqslant \aleph_{1}$.

- Let $\pi \geqslant \lambda^{+} \geqslant \aleph_{2}$ be an uncountable regular cardinal such that $\pi^{\omega}=\pi$.

- Let $S \subseteq\left(S_{\omega}^{\pi}\right)^{L}$ be a stationary set in $\mathbb{L}$;

- Let $\bar{S}=\left\langle\eta_{\alpha}: \alpha \in S\right\rangle$, where each $\eta_{\alpha}$ is an increasing cofinal sequence in $\alpha$ of order type $\omega$ (i.e., a $\pi$-club guessing sequence ${ }^{1}$ ). We are guaranteed the existence of this club guessing sequence because $\pi \geqslant \aleph_{2}$.

We define our first pair of trees.

\section{Definition 3.3.}

- Let

$$
I_{0}=I(\pi, \bar{S})
$$

be an ordered tree in $K_{t r}^{\omega}(\pi)$, with cardinality $\left|I_{0}\right|=\pi$, having universe

$$
{ }^{<\omega} \pi \cup\left\{\eta_{\alpha}: \eta_{\alpha} \in \bar{S}\right\} \subset{ }^{\leqslant \omega} \pi,
$$

where the relations are as always on ordered trees.

- Let

$$
I_{1}=I(\pi,\langle\rangle)={ }^{<\omega} \pi
$$

The tree $I_{1}$ is also in $K_{t r}^{\omega}(\pi)$, and $\left|I_{1}\right|=\pi$.

3.1.2. The second generation of trees. Now, we define the domains of our next generation of trees. This next generation is needed so that we have nonisomorphic $L_{\infty \pi^{-}}$equivalent trees in $K_{t r}^{\omega}(\pi)$ with certain further useful properties (see [HT91] Definition 8.19 and Lemma 8.20, and [HHR04] Lemma 7.29). The non-isomorphism of the pair of trees $I_{0}$ and $I_{1}$ is easy to detect. We therefore need a new pair of trees where this non-isomorphism is more "obscured". This construction is originally due to Shelah [She87].

\footnotetext{
${ }^{1}$ For a definition, see p. 442 of [Jec03]
} 
Let

- $\operatorname{LEX}\left({ }^{<\omega} \pi\right)$ be a linear order with universe ${ }^{<\omega} \pi$, ordered lexicographically.

- $\mathrm{OT}_{\pi}\left({ }^{<\omega} \pi\right)$ be a linear (well) order with universe ${ }^{<\omega} \pi$, ordered with order type $\pi$.

- $\theta=\mathrm{OT}_{\pi}\left({ }^{<\omega} \pi\right) \cdot \operatorname{LEX}\left({ }^{<\omega} \pi\right)$ be the product of the linear orders $\mathrm{OT}_{\pi}\left({ }^{<\omega} \pi\right)$ and $\operatorname{LEX}\left({ }^{<\omega} \pi\right)$ whose universe is $\mathrm{OT}_{\pi}\left({ }^{<\omega} \pi\right) \times \operatorname{LEX}\left({ }^{<\omega} \pi\right)$.

Let

$$
\begin{gathered}
\bar{I}_{0}=\left\langle I_{0} \cap \leqslant \omega \alpha: \alpha<\pi\right\rangle, \\
\bar{I}_{1}=\left\langle I_{1} \cap \leqslant \omega \alpha: \alpha<\pi\right\rangle=\left\langle{ }^{<\omega} \pi \cap \leqslant \omega \alpha: \alpha<\pi\right\rangle=\left\langle{ }^{<\omega} \alpha: \alpha<\pi\right\rangle
\end{gathered}
$$

be $\pi$-filtrations of $I_{0}$ and $I_{1}$, respectively.

The filtrations are used in Definition 3.5 to ensure that the trees we build are not "too similar".

Lemma 3.4 ([HHR04] Lemma 7.24 or [HT91] Lemma 8.17). Let $\pi$ be a cardinal. Suppose $\operatorname{LEX}\left({ }^{<\omega} \pi\right)$ is as above. Then there is $E \subseteq \operatorname{LEX}\left({ }^{<\omega} \pi\right)$ of cardinality $\pi$ such that for any $a, b \in E$ there is an automorphism $g_{a, b}$ of $\operatorname{LEX}\left({ }^{<\omega} \pi\right)$ which maps a to $b$.

Let $E \subseteq \operatorname{LEX}\left({ }^{<\omega} \pi\right)$ be as given by Lemma 3.4. Fix $c \in E$. Let $g$ be a bijection $g:\left\{R: R \in \operatorname{rng}\left(\bar{I}_{0}\right) \cup \operatorname{rng}\left(\bar{I}_{1}\right)\right\} \longrightarrow E \backslash\{c\}$.

Definition 3.5. Let $J_{0}=J\left(c, g, \bar{I}_{0}, \bar{I}_{1}\right)$ have a universe consisting of functions $\eta \in{ }^{\leqslant \omega} \theta$, such that one of the following holds

(1) $\eta \in{ }^{<\omega} \theta$ (in other terms, $\eta \in \operatorname{DOM}_{n}$ for some $n \in \omega$; i.e. $\eta$ is of finite length);

(2) there is $s \in I_{0}$ such that $\operatorname{dom}(s)=\omega$, and for all $n<\omega$,

$$
\eta(n)=\left\langle s \uparrow_{(n+1)}, c\right\rangle ;
$$

(3) there are $m<\omega, R \in \operatorname{rng}\left(\bar{I}_{0}\right) \cup \operatorname{rng}\left(\bar{I}_{1}\right)$, and $s \in R$ with $\operatorname{dom}(s)=\omega$ such that for all finite $n \geqslant m, \eta(n)=\left\langle s \uparrow_{(n+1)}, g(R)\right\rangle$.

Let $J_{1}=J\left(c, g, \bar{I}_{1}, \bar{I}_{0}\right)$ be defined analogously. Note that $J_{1}$ differs from $J_{0}$ only in that $J_{1}$ does not have any members satisfying condition 2 of the definition.

The trees $J_{0}$ and $J_{1}$ are isomorphic to ordered trees in $K_{t r}^{\omega}(\theta)$, so we assume that $J_{0}, J_{1} \in K_{t r}^{\omega}(\theta)$.

Lemma 8.20 of [HT91] establishes that $J_{0}$ and $J_{1}$ are $\mathcal{L}_{\infty \pi}$-equivalent.

3.1.3. The third generation: a quasi-order. At this point in the construction, we can lose the $<_{\text {lex }}$ ordering on $J_{i}$, since we do not need it for the primary model construction that follows. Indeed, we could have used a different construction in the second generation that did not feature $<_{l e x}$. However, we chose to take advantage of the existing construction from [She87] to save some effort.

Let $K_{i}=\mathscr{P}^{<\omega}\left(J_{i}\right)$ be the set of all finite subsets of $J_{i}, i=0,1$, respectively.

We define relations as in [HS98]. Let $u, v \in K_{i}$. We define the "minimum" set of initials $\operatorname{MinSetIn}(u, v)$ to be the largest set $X$ such that: 
(1) $X \subseteq\{\operatorname{MaxInSg}(\zeta, \eta): \zeta \in u, \eta \in v\}$;

(2) if $\eta_{i}, \eta_{j} \in X$ and $\eta_{i}$ is an initial segment of $\eta_{j}$, then $\eta_{i}=\eta_{j}$.

Note that

$\operatorname{MinSetIn}(u, u)=\{\zeta \in u: \neg \exists \eta \in u(\zeta$ is a proper initial segment of $\eta)\}$.

The elements of $K_{i}$ are ordered by $<^{K}: u<^{K} v$ iff for every $\zeta \in u$ there is $\eta \in v$ such that $\zeta$ is an initial segment of $\eta$. In other terms,

$$
u \leq{ }^{K} v \text { iff } \operatorname{MinSetIn}(u, v)=\operatorname{MinSetIn}(u, u) .
$$

Note that $\left(K_{i},<^{K}\right)$ cannot have infinite descending chains.

Definition 3.6. We call $s \in K_{i}$ semi-good if $s$ is an antichain with regard to the $\lessdot$ relation in $J_{i}$.

Denote by $\bar{s}$ the downwards closure of $s$. We say that $r \in K_{i}$ is good if it is downwards closed and $r \subset \bar{s}$, where $s$ is semi-good. We denote by $G\left(K_{i}\right)$ the collection of good elements of $K_{i}$.

3.2. Building the models: putting fat on the trees. We will base a primary model construction based on the trees $J_{i}$, using the quasi-order $K_{i}$.

3.2.1. Cardinal assumptions. Recall that we assume in this section that we work within $\mathbf{m}_{\mathbf{l}}$, a strictly stable homogeneous monster model of cardinality $\|\mathbf{m}\|=.\mu$. We let $\lambda(\mathbf{m})$ be the first cardinal in which $\mathbf{m}$ is stable, and we let $\lambda=\lambda_{r}(\mathbf{m})$ be the first regular cardinal $\geqslant \lambda\left(\mathbf{m}_{\mathbf{l}}\right)$. By our assumption that $\boldsymbol{m}$ is strictly stable, $\kappa\left(\mathbf{m}_{\mathbf{l}}\right) \neq \omega$ (see 3.7 below). Thus, $\aleph_{1} \leqslant \kappa\left(\mathbf{m}_{\mathbf{l}}\right) \leqslant \lambda\left(\mathbf{m}_{\mathbf{l}}\right) \leqslant \lambda$. Further, let $\pi$ be a regular cardinal such that $\pi^{\omega}=\pi$ and $\lambda<\pi<\mu$. Thus $\pi \geqslant \aleph_{2}$. This $\pi$ is the size of the models that we will be building, and is the cardinal upon which our trees have been built.

We proceed with the construction similarly to [HS98].

3.2.2. An initial $\omega$-sequence of models. We restate the following lemma, which provides the seed for our construction:

Lemma 3.7 (Lemma 5.1 [HS00]). The following are equivalent:

(1) $\mathrm{m}$ is not superstable.

(2) $\kappa\left(\mathbf{m}_{\mathbf{l}}\right) \neq \omega$.

(3) There is an increasing sequence $\mathscr{A}_{n}, n<\omega$ of $\mathbf{F}_{\lambda(\mathrm{m})}^{\mathrm{m}_{\mathrm{m}}}$-saturated models and an element a such that for all $n<\omega, a \succeq_{\mathscr{A}_{n}} \mathscr{A}_{n+1}$.

Remark 3.8. The sequence $\mathscr{A}_{n}, n<\omega$ in Lemma 3.7 can be chosen to consist of models of size $\lambda$.

Proof. Let $\mathscr{A}_{n}, n<\omega$ be the sequence of models given by Lemma 3.7. It is easy to find such models that are quite large.

Each $\mathscr{A}_{n}$ is $\mathbf{F}_{\lambda}^{\mathrm{m}}$-saturated, and hence strongly $\mathbf{F}_{\kappa(\mathbf{m}}^{\mathrm{m}} \mathrm{m}^{\text {-saturated by Lemma }}$ 1.9(iv) of [HS00]. Thus, by the monotonicity given by Lemmas 1.2(vi) and 1.13, and the 
proof of Lemma 3.2(iii) of that same paper, there exist $B_{n} \subset \mathscr{A}_{n}$ an increasing sequence of sets of size $<\kappa\left(\mathbf{m}_{\mathbf{e}}\right)$ such that

$$
a \underset{B_{n}}{\perp} \mathscr{A}_{n}
$$

We also have that $a\rfloor_{B_{i}} \mathscr{A}_{i+1}$. By the finite character of independence in our setting (Corollary 3.5(i) of [HS00]), there exist finite $b_{n+1} \in \mathscr{A}_{n+1}$ that witness $a £_{B_{n}} \mathscr{A}_{n+1}$ such that

$$
a \succeq b_{B_{n}} .
$$

Choose $\mathbf{F}_{\lambda}^{\mathrm{m}}$-saturated models $\mathscr{C}_{n}$ of size $\lambda$ so that $B_{n} \subset \mathscr{C}_{n} \subset \mathscr{A}_{n}$ and $b_{n+1} \in$ $\mathscr{C}_{n+1}$. We can do this by Theorem 3.14 of [HS00].

We claim that $\left(\mathscr{C}_{n}\right)_{n<\omega}$ satisfy the requirements of Lemma 3.7: Assume the contrary, that $a \downarrow_{\mathscr{C}_{n}} \mathscr{C}_{n+1}$. Since $a \downarrow_{B_{n}} \mathscr{A}_{n}, a \downarrow_{B_{n}} \mathscr{C}_{n}$ by monotonicity. By transitivity and monotonicity, $a \downarrow_{B_{n}} \mathscr{C}_{n+1}$. Finally, monotonicity gives us

$$
a \underset{B_{n}}{\downarrow} b_{n+1},
$$

and hence a contradiction.

Construction Element. Thus, fix $\left(\mathscr{A}_{j}\right)_{j \leqslant \omega}$, a sequence of $\left.\mathbf{F}_{\lambda(\mathrm{m})}^{\mathrm{m}}\right)^{\text {-saturated mod- }}$ els of size $\lambda$, and an element $a$ with the properties as in Lemma 3.7.

Construction Element. Let $\mathscr{A}_{\omega}$ be a $\mathbf{F}_{\lambda_{r}(\mathrm{~m})}^{\mathrm{m}}$-primary model over

$$
a \cup \bigcup_{i<\omega} \mathscr{A}_{i}
$$

the existence of which is guaranteed by Theorem 5.3 of [HS00] (proof is in [She70]).

\subsubsection{The construction.}

Construction Element. For all $\eta \in \pi^{\leqslant \omega}$, using analogous reasoning to that found in Section 1 of [Hyt97a] (discussion of which begins after Theorem 1.15 and continues through the proof of Lemma 1.17 of that paper), we define models $\mathscr{A}_{\eta}$ such that

- for all $\eta \in \leqslant \omega \pi$, there is an automorphism $f_{\eta}$ of $\boldsymbol{m}$ such that

$$
f_{\eta}\left(\mathscr{A}_{\text {length }(\eta)}\right)=\mathscr{A}_{\eta} ;
$$

- if $\eta$ is an initial segment of $\zeta$, then

$$
f_{\zeta} \Upsilon_{\mathscr{A}_{\text {length }(\eta)}}=f_{\eta} \Upsilon_{\mathscr{A}_{\text {length }(\eta)}} ;
$$


- if $\eta \in{ }^{<\omega} \pi, \alpha \in \pi$, and $X$ is the set of those $\eta \in{ }^{\leqslant \omega} \pi$ such that $\eta \smile(\alpha)$ is an initial segment of $\zeta$, then

$$
\bigcup_{\zeta \in X} \mathscr{A}_{\zeta} \underset{\mathscr{A}_{\eta}}{\downarrow} \bigcup_{\zeta \in\left(\leqslant \omega_{\pi \backslash X)}\right.} \mathscr{A}_{\zeta}
$$

- for all $\eta \in{ }^{\omega} \pi$, we let $a_{\eta}=f_{\eta}(a)$.

We recall a definition which will allow us to carry out the construction in an orderly and controlled manner.

Definition 3.9 (Definition 3 of [HS98]). Assume $J \subseteq \leqslant \omega \pi$ is closed under initial segments and $K=\mathscr{P}^{<\omega}(J)$. Let $\Sigma=\left\{A_{u}: u \in K\right\}$ be an indexed family of subsets of $\mathrm{m}$ of cardinality $<\mu$. We say that $\Sigma$ is strongly independent if

(1) for all $u, v \in K, u \leq^{K} v \rightarrow A_{u} \subseteq A_{v}$;

(2) if $u, u_{i} \in K, i<n \in \omega$, and $B \subseteq \bigcup_{i<n} A_{u_{i}}$ has cardinality $<\pi$, then there is an automorphism $f=f_{\left(u, u_{0}, \ldots, u_{n-1}\right)}^{\Sigma, B}$ of $\boldsymbol{m}$ such that $f \Upsilon_{\left(B \cap A_{u}\right)}=i d_{B \cap A_{u}}$, and $f\left(B \cap A_{u_{i}}\right) \subseteq A_{\operatorname{MinSetIn}\left(u, u_{i}\right)}$.

Construction Element. Define

$$
A_{u}^{i}=\bigcup_{\eta \in u} \mathscr{A}_{\eta}
$$

for $u \in K_{i}$.

We can now apply Lemma 6 of [HS98] to find that $\left\{A_{u}^{i}: u \in K_{i}\right\}$ is strongly independent.

Construction Element. We apply Lemma 4 of [HS98] to $\left\{A_{u}^{i}: u \in K_{i}\right\}$, and so find models $\mathscr{A}_{u}^{i} \preccurlyeq \mathbf{m}, u \in K_{i}$ which satisfy the following properties:

(1) For all $u, v \in K_{i}, u \leq{ }^{K} v$ implies $\mathscr{A}_{u}^{i} \subseteq \mathscr{A}_{v}^{i}$;

(2) for all $u \in K_{i}, \mathscr{A}_{u}^{i}$ is $\mathbf{F}_{\lambda_{r}\left(\mathbf{m}_{\mathbf{c}}\right)}^{\mathrm{m}}$-primary over $A_{u}^{i}$. This implies that $\bigcup_{u \in K_{i}} \mathscr{A}_{u}^{i}$ is a model.

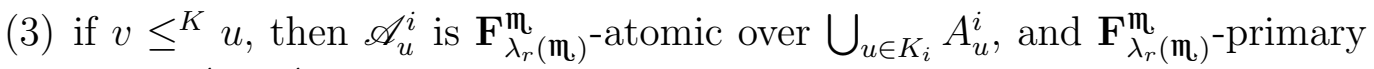
over $\mathscr{A}_{v}^{i} \cup A_{u}^{i}$.

(4) Note further that if $J^{\prime} \subseteq J_{i}$ is closed under initial segments, and $u \in$ $P^{<\omega}\left(J^{\prime}\right)$, then the union $\bigcup_{v \in P^{<\omega}\left(J^{\prime}\right)} \mathscr{A}_{v}$ is $\mathbf{F}_{\lambda_{r}(\mathrm{~m} .)^{-}}^{\mathrm{m}_{\mathrm{l}}}$-constructible over $\mathscr{A}_{u} \cup \bigcup_{v \in P<\omega\left(J^{\prime}\right)} A_{v}$.

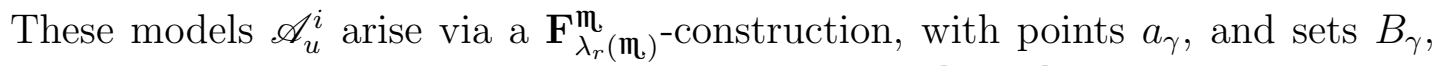
$\gamma<\alpha$ chosen appropriately. See proof of Lemma 4, [HS98] for full details.

In addition, note that by the proof of [HS98] Lemma 4 (Claim), the families of models $\left\{\mathscr{A}_{u}^{i}: u \in K_{i}\right\}$, where $i=0$ or $i=1$ are strongly independent.

Construction Element. Denote by

$$
\mathscr{A}^{J_{i}}=\bigcup_{u \in K_{i}} \mathscr{A}_{u}^{i}
$$

the resulting constructed models given by Lemma 4 of [HS98]. 
3.3. Non-isomorphism when symmetric difference of $S$-invariants is stationary. We prove some general facts concerning models built as above on arbitrary trees $J, J^{\prime} \subseteq \pi^{\leqslant \omega}$. We will later apply these results to $J_{0}$ and $J_{1}$.

Definition 3.10. Denote by $I_{N S^{\pi}}$ be the ideal of non-stationary sets on $\pi$.

For $J \subseteq \pi^{\leqslant \omega}$, let $J^{\alpha}=J \cap \alpha^{\leqslant \omega}$.

For $K=\mathscr{P} \leqslant \omega(J)$, let $K^{\alpha}=\mathscr{P} \leqslant \omega\left(J^{\alpha}\right)$.

Define the $S$-invariant of $J$ to be:

$$
S(\bar{J})=\left\{\delta: \exists \eta \in J^{\delta}\left(\eta \notin \bigcup_{\alpha<\delta} J^{\alpha}\right)\right\} \text { modulo } I_{N S^{\pi}} .
$$

Lemma 3.11. Let $\mathscr{A}^{J}$ and $\mathscr{A}^{J^{\prime}}$ be models constructed as above for trees $J, J^{\prime} \subseteq$ $\pi^{\leqslant \omega}$. Assume that $S(J) \triangle S\left(J^{\prime}\right)=\left(S(J) \backslash S\left(J^{\prime}\right)\right) \cup\left(S\left(J^{\prime}\right) \backslash S(J)\right)$ is stationary. Then $\mathscr{A}^{J} \not \mathscr{A}^{J^{\prime}}$.

Proof. We follow Lemma 8 of [HS98].

Assume for a contradiction that $f: \mathscr{A}^{J} \longrightarrow \mathscr{A}^{J^{\prime}}$ is an isomorphism.

Let $\bar{J}=\left(J^{\alpha}\right)_{\alpha<\pi}, \bar{J}^{\prime}=\left(J^{\prime \alpha}\right)_{\alpha<\pi}$. Let $K=\mathscr{P} \leqslant \omega(J), K^{\prime}=\mathscr{P} \leqslant \omega\left(J^{\prime}\right)$, and let $K^{\alpha}=\mathscr{P} \leqslant \omega\left(J^{\alpha}\right), K^{\prime \alpha}=\mathscr{P} \leqslant \omega\left(J^{\prime \alpha}\right)$.

Let $\mathscr{A}_{J}^{\alpha}=\bigcup_{s \in G\left(K^{\alpha}\right)} \mathscr{A}_{s}$, where $G\left(K^{\alpha}\right)$ is the collection of good elements of $K^{\alpha}$, as defined in Definition 3.6.

We can find $\alpha$ and $\alpha_{i}, i<\omega$ such that

- $\eta=\left(\alpha_{i}\right)_{i<\omega}$ is strictly increasing for all $i<\omega$,

- $\alpha=\bigcup_{i<\omega} \alpha_{i} \in S(J) \triangle S\left(J^{\prime}\right)$,

and

$$
f \Gamma_{\mathscr{A}_{J}^{\alpha}}: \mathscr{A}_{J}^{\alpha} \stackrel{\cong}{\longrightarrow} \mathscr{A}_{J^{\prime}}^{\alpha}
$$

are isomorphisms.

$$
f \uparrow_{\mathscr{A}_{J}^{\alpha_{i}}}: \mathscr{A}_{J}^{\alpha_{i}} \stackrel{\cong}{\longrightarrow} \mathscr{A}_{J^{\prime}}^{\alpha_{i}}, \forall i<\omega
$$

Without loss of generality, we can assume that $\alpha \in S(J) \backslash S\left(J^{\prime}\right)$ and thus that $\eta \in J \backslash J^{\prime}$.

Claim 3.11.1.

$$
a_{\eta} \underset{\mathscr{A}_{J}^{\alpha_{i}}}{\longleftarrow} \mathscr{A}_{J}^{\alpha_{i+1}}
$$

Recall from the construction that

$$
a_{\eta} \underset{\mathscr{A}_{\eta \uparrow_{i}}}{\longleftarrow} \mathscr{A}_{\eta \uparrow_{i+1}} \text {. }
$$

Since $\mathscr{A}_{\eta \uparrow_{i}} \subset \mathscr{A}_{J}^{\alpha_{i}}$ and $\mathscr{A}_{\eta \uparrow_{i+1}} \subset \mathscr{A}_{J}^{\alpha_{i+1}}$, and $\mathscr{A}_{\eta \uparrow_{i+1}} \not \subset \mathscr{A}_{J}^{\alpha_{i}}$, by monotonicity (Lemma 3.2 (i), [HSO0]), we have

$$
a_{\eta} \underset{\mathscr{A}_{\eta \uparrow_{i}}}{\downarrow} \mathscr{A}_{\eta \uparrow_{i+1}} \Rightarrow a_{\eta} \underset{\mathscr{A}_{\eta \uparrow_{i}}}{\downarrow} \mathscr{A}_{J}^{\alpha_{i+1}}
$$


Claim $\left(3.11 .1^{*}\right)$. Thus, to prove Claim 3.11.1, it is enough to show that

$$
a_{\eta} \underset{\mathscr{A}_{J}^{\alpha_{i}}}{£} \mathscr{A}_{\eta \uparrow_{i+1}} .
$$

Assume for a contradiction that $a_{\eta} \downarrow_{\mathscr{A}_{J}^{\alpha_{i}}} \mathscr{A}_{\eta \uparrow_{i+1}}$.

Claim 3.11.2.

$$
a_{\eta} \underset{\mathscr{A}_{J}^{\alpha_{i}}}{\downarrow} \mathscr{A}_{\eta \uparrow_{i+1}} \Rightarrow \mathscr{A}_{J}^{\alpha_{i}} \underset{\mathscr{A}_{\eta \Gamma_{i}}}{\downarrow} \mathscr{A}_{\eta \Gamma_{i+1}} .
$$

By assumption, $a_{\eta} \downarrow_{\mathscr{A}_{J}^{\alpha_{i}}} \mathscr{A}_{\eta \uparrow_{i+1}}$. This implies that $\mathscr{A}_{\eta \uparrow_{i+1}} \downarrow_{\mathscr{A}_{J}^{\alpha_{i}}} a_{\eta}$. We get this symmetry by using monotonicity to find that $a_{\eta} \downarrow_{\mathscr{A}_{J}^{\alpha_{i}}} \bar{b}$ for any finite $\bar{b} \in \mathscr{A}_{\eta \uparrow_{i+1}}$. Then, since $\mathscr{A}_{J}^{\alpha_{i}}$ is $\mathbf{F}_{\lambda(\mathrm{m})}^{\mathrm{m}}$-saturated by construction ([HS98]), and hence strongly $\mathbf{F}_{\kappa(\mathrm{m})}^{\mathrm{m}_{\mathrm{H}}}$-saturated, by Lemma 3.6 of $[\mathrm{HS} 00], \bar{b} \downarrow_{\mathscr{A}_{J}^{\alpha_{i}}} a_{\eta}$. Since this is true for all $\bar{b} \in \mathscr{A}_{\eta \uparrow_{i+1}}$, we get

$$
\mathscr{A}_{\eta \uparrow_{i+1}} \underset{\mathscr{A}_{J}^{\alpha_{i}}}{\downarrow} a_{\eta} .
$$

Now, assume for a contradiction that $\mathscr{A}_{J}^{\alpha_{i}} \downarrow_{\mathscr{A}_{\eta \uparrow_{i}}} \mathscr{A}_{\eta \uparrow_{i+1}}$. By a similar symmetry argument, $\mathscr{A}_{\eta \uparrow_{i+1}} \downarrow_{\mathscr{A}_{\eta \uparrow_{i}}} \mathscr{A}_{J}^{\alpha_{i}}$.

Thus, we have

$$
\mathscr{A}_{\eta \uparrow_{i+1}} \underset{\mathscr{A}_{J}^{\alpha_{i}}}{\downarrow} a_{\eta} \text { and } \mathscr{A}_{\eta \uparrow_{i+1}} \underset{\mathscr{A}_{\eta \uparrow_{i}}}{\downarrow} \mathscr{A}_{J}^{\alpha_{i}}
$$

In addition, by [HS98] Lemma 3.2 (iii), we have $a_{\eta} \downarrow_{\mathscr{A}_{J}^{\alpha_{i}}} \mathscr{A}_{J}^{\alpha_{i}}$. We can thus apply [HS98] Lemma 3.8 (iii) to find that

$$
\mathscr{A}_{\eta \uparrow_{i+1}} \underset{\mathscr{A}_{\eta \uparrow_{i}}}{\perp} a_{\eta} \cup \mathscr{A}_{J}^{\alpha_{i}}
$$

By monotonicity and symmetry, we get $a_{\eta} \downarrow_{\mathscr{A}_{\eta \Gamma_{i}}} \mathscr{A}_{\eta \uparrow_{i+1}}$, a contradiction.

$\nabla_{\text {Claim } 3.11 .2}$

Thus, with our assumptions so far, we have $\mathscr{A}_{\eta \uparrow_{i+1}} £_{\mathscr{A}_{\eta \uparrow_{i}}} \mathscr{A}_{J}^{\alpha_{i}}$. We now show that this dependence causes a contradiction.

Since $\mathscr{A}_{\eta\rangle_{i}}$ is sufficiently saturated, by [HS00] Corollary 3.5 (i), there is $c \in \mathscr{A}_{J}^{\alpha_{i}}$ such that

$$
\mathscr{A}_{\eta \uparrow_{i+1}} \underset{\mathscr{A}_{\eta \uparrow_{i}}}{\downarrow} c .
$$

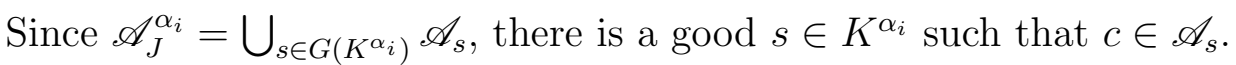

Now, let $r=\left\{\eta \uparrow_{j}: j \leqslant i+1\right\}$. Then, $r$ is good and $r \cap J^{\alpha_{i}}=\left\{\eta \uparrow_{j}: j \leqslant i\right\}$. Without loss of generality, we can assume that $\eta \uparrow_{i} \in s$, since $\mathscr{A}_{s}$ cannot get smaller with this assumption. 
However, by strong independence (see [HS98]), $\mathscr{A}_{r} \downarrow_{\mathscr{A}_{r \cap s}} \mathscr{A}_{s}$, which by definition, written otherwise

$$
\mathscr{A}_{\eta \uparrow_{i+1}} \underset{\mathscr{A}_{\eta \uparrow_{i}}}{\downarrow} \mathscr{A}_{s} .
$$

This gives a contradiction since $c \in \mathscr{A}_{s}$.

$\nabla_{\text {Claim } 3.11 .1}$

Thus, there is $u \in K^{\prime}$ such that for all $\left.i<\omega, \mathscr{A}_{u}\right\rfloor_{\mathscr{A}_{J^{\prime}}^{\alpha_{i}}} \mathscr{A}_{J^{\prime}}^{\alpha_{i+1}}$. However, since $\alpha \notin S\left(J^{\prime}\right)$, this contradicts Lemma 7 (ii) of [HS98]. Since the notation we use here is rather different from that in [HS98], note that we can find a model with the properties of what is in [HS98] defined by $\mathcal{A}_{v}$ in $\mathscr{A}_{J^{\prime}}^{\alpha_{i}}$. Recall that $\mathscr{A}_{J^{\prime}}^{\alpha_{i+1}}$ can be written as a union of appropriate models as in the notation found in [HS98].

$\square_{3.11}$

Corollary 3.12. Let $\mathscr{A}^{J}$ and $\mathscr{A}^{J^{\prime}}$ be models constructed as above for trees $J, J^{\prime} \subseteq \pi^{\leqslant \omega}$. Assume that $S(J)=S \subset S_{\omega}^{\pi}$ and $S\left(J^{\prime}\right)=\emptyset$, thus $S(J) \triangle S\left(J^{\prime}\right)=S$ is stationary. Then $\mathscr{A}^{J} \neq \mathscr{A}^{J^{\prime}}$ in any cardinal- and $\mathscr{P}\left(\lambda_{r}(\mathbf{m})\right)$-preserving extension of the universe where the symmetric difference $S(J) \triangle S\left(J^{\prime}\right)$ remains stationary.

Notice that the proof of Lemma 3.11 is in ZFC. In particular, the notion of independence is absolute for models where no small (of size $<\lambda_{r}\left(\mathbf{m}_{\mathbf{}}\right.$ )) subsets are added. Thus, two models $\mathscr{A}_{J}$ and $\mathscr{A}_{J^{\prime}}$ which are non-isomorphic in the ground model remain non-isomorphic in any cardinal- and $\mathscr{P}\left(\lambda_{r}(\mathbf{m})\right)$-preserving extension of the universe where the symmetric difference $S(J) \triangle S\left(J^{\prime}\right)$ remains stationary.

It is easy to see that $S\left(J_{0}\right)=S$ and $S\left(J_{1}\right)=\emptyset$. Thus, we can apply the previous lemma to find that in $\mathbb{L}, \mathscr{A}_{J_{0}} ¥ \mathscr{A}_{J_{1}}$.

\subsection{Isomorphism of the models when $S$ is killed.}

Theorem 3.13. Assume that in some extension of the set-theoretic universe which preserves cardinals and $\mathscr{P}\left(\lambda_{r}\left(\mathbf{m}_{\mathbf{}}\right)\right), J_{0} \cong J_{1}$. Then in that extension $\mathscr{A}_{J_{0}} \cong \mathscr{A}_{J_{1}}$.

Proof. Assume that $F: J_{0} \longrightarrow J_{1}$ is an isomorphism. We aim to find an isomorphism between $\mathscr{A}_{J_{0}}$ and $\mathscr{A}_{J_{1}}$.

We proceed by induction on good elements of $K_{0}$ along the ordering $\leq^{K}$ by building elementary maps $G_{u}, u \in K_{0}$. We ensure in this induction that if $u_{i} \leq{ }^{K} u_{j}$ and $u_{j} \mathbb{L}^{K} u_{i}$ then $G_{u_{i}}$ is constructed before $G_{u_{j}}$.

Base case: isomorphism for the first level of the tree $G_{0}$ : For all $u \in$ $K_{0}=\mathscr{P}<\omega\left(J_{0}\right)$, let $F(u)=\{F(\eta): \eta \in u\}$. For $\eta \in J_{0}$, let $G_{0} \Upsilon_{\mathscr{A}_{\eta}}=f_{F(\eta)} \circ f_{\eta}^{-1}$, where the $f_{\eta}$ are as defined in Section 3.2.3. Thus,

$$
G_{0}: \bigcup_{\eta \in J_{0}} \mathscr{A}_{\eta} \longrightarrow \bigcup_{\eta \in J_{1}} \mathscr{A}_{\eta}
$$


Claim 3.13.1. The function $G_{0}$, which maps one strongly independent family to the other, is elementary.

We prove the claim by induction on good elements $s \in K_{0}$ along the ordering $\leq$. Denote by $G_{0}^{\eta}=f_{F(\eta)} \circ f_{\eta}^{-1}$, and by $G_{0}^{s}=\bigcup_{\xi \in s} G_{0}^{\xi}$, for $s \in G\left(J_{0}\right)$.

By construction, $G_{0}^{\eta}, \eta \in J_{0}$ is elementary.

Now, assume that $G_{0}^{s}$ has been shown to be elementary. We wish to show that $G_{0}^{s^{\prime}}$ for $s^{\prime} \geq^{K} s$ is also elementary. Our ordering of $G\left(J_{0}\right)$ implies that it is enough to consider $s^{\prime}=s \cup\{\eta\}$ for some $\eta \in J_{0}$. We thus have two cases: $\eta \in \pi^{<\omega}$ or $\eta \in \pi^{\omega}$. The arguments for both are similar.

If $\eta \in \pi^{<\omega}$, denote by $\eta^{-}=\eta \uparrow_{(\text {length }(\eta)-1)}$. If $\eta \in \pi^{\omega}$ is an infinite branch, then we can then find $i<\omega$ such that $\forall \xi \in s, \xi \ngtr \eta \uparrow_{i}$. Denote by $\eta^{-}=\eta \uparrow_{i-1}$ ).

Since we are working in a homogeneous monster model $\mathbf{m}$, we can assume without loss of generality that $G_{0}^{s} \uparrow_{A_{s}}=i d_{A_{s}}$.

In addition, we know from the construction that

$$
\operatorname{tp}_{\mathrm{m}}\left(\mathscr{A}_{\eta} / \mathscr{A}_{\eta^{-}}\right)=\operatorname{tp}_{\mathrm{m}}\left(G_{0}^{\eta}\left(\mathscr{A}_{\eta}\right) / \mathscr{A}_{\eta^{-}}\right) \text {, }
$$

because $G_{0}^{\eta}$ is elementary and $G_{0}^{\eta} \Upsilon_{\mathscr{A}_{\eta}}=i d$. We thus want to show that

$$
\operatorname{tp}_{\mathrm{m}}\left(\mathscr{A}_{\eta} / A_{s}\right)=\operatorname{tp}_{\mathrm{m}}\left(G_{0}^{\eta}\left(\mathscr{A}_{\eta}\right) / A_{s}\right) \text {. }
$$

Since $\mathscr{A}_{\eta}$ is $\mathbf{F}_{\lambda(\mathrm{m} .)}^{\mathrm{m}}$-saturated, these types are stationary. Therefore, by definition of stationarity (Def. 3.3. [HS00]) it is enough to show that

$$
\mathscr{A}_{\eta} \underset{\mathscr{A}_{\eta^{-}}}{\perp} A_{s} \text { and } G_{0}^{\eta}\left(\mathscr{A}_{\eta}\right) \underset{\mathscr{A}_{\eta^{-}}}{\perp} A_{s} \text {. }
$$

However, note that $\eta^{-} \in s=f(s)$, thus we have the independence by construction, and so the embedding is elementary.

$\nabla_{\text {Claim } 3.13 .1}$

Before we continue with the next step of the induction, we give some notation and reminders. Denote $\mathscr{A}_{\{\eta\}}=\mathscr{A}_{\eta}$. Recall that by the construction, $A_{u}=$ $\bigcup_{\eta \in u} \mathscr{A}_{\eta}$ for $u \in K_{i}$, and $\mathscr{A}_{u}$ is $\mathbf{F}_{\lambda_{r}(\mathrm{~m})}^{\mathrm{m}}$-prime over $A_{u}$.

Ultimately, we aim to build an isomorphism $G: \mathscr{A}^{J_{0}} \longrightarrow \mathscr{A}^{J_{1}}$ such that $G \uparrow_{\mathscr{A}_{\eta}}=$ $i d_{\mathscr{A}_{\eta}}$ for all $\eta \in J_{i}$. Since $\mathscr{A}^{J_{i}}=\bigcup_{u \in K_{i}} \mathscr{A}_{u}^{i}$, it is enough to construct $G_{u}: \mathscr{A}_{u}^{0} \longrightarrow$ $\mathscr{A}_{u}^{1}$ such that if $t \leq{ }^{K} u$, then $G_{t} \subseteq G_{u}$. If we can show that $\bigcup_{t \leq K_{u}} G_{t}$ is elementary, then using homogeneity of $\mathbf{m}_{\mathbf{l}}$, we can find the desired isomorphism $G_{u}$. The full isomorphism will then be $G=\bigcup_{u \in G\left(K_{i}\right)} G_{u}$.

Inductive step: Assume we have shown that for all $t \lesseqgtr u, G_{t}$ are isomorphisms. We build an isomorphism $G_{u}: \mathscr{A}_{u}^{0} \longrightarrow \mathscr{A}_{u}^{1}$.

Claim 3.13.2. The function $\bigcup_{t<{ }^{K} u} G_{t}$ is elementary.

We assume for a contradiction that the inductive step fails at some point. Let $u$ be the $\leq^{K}$-smallest such that $\bigcup_{t \leq K_{u}} G_{t}=G^{*}$ is not elementary.

This failure of elementariness is witnessed by some set of finitely many points $a_{0}, \ldots, a_{m} \in \bigcup_{t \leq K^{K} u} \mathscr{A}_{t}$. Then, in particular, $G^{*} \uparrow_{\left\{a_{0}, \ldots, a_{m}\right\}}$ is not elementary. 
Subclaim 3.13.2.1. The points $a_{0}, \ldots, a_{m}$ can be replaced with tuples $\bar{a}_{i}$, $i=0, \ldots n$ which appear all at once at a given step in the construction, that is, $\bar{a}_{i} \in \mathscr{A}_{t_{i}}$ and $\bar{a}_{i} \cap \bigcup_{t<{ }^{K} t_{i}} \mathscr{A}_{i}=\emptyset$.

Consider $a_{0}, \ldots, a_{m}$. For all $i \leqslant m$, there is $t^{i}<^{K} u$ such that $a_{i} \in \mathscr{A}_{t^{i}}$ $\bigcup_{t<{ }^{K} t^{i}} \mathscr{A}_{t}$. Let $\left\{t_{0}, \ldots, t_{n}\right\}$ be an enumeration of the $t^{i}$ so that $t_{i} \neq t_{j}$ if $i \neq j$ (i.e., we get rid of repetitions). In addition, we can assume without loss of generality that $t_{n}$ is maximal in $\left\{t_{0}, \ldots, t_{n}\right\}$ with respect to the ordering $\leq^{K}$.

Define $\bar{a}_{i}=\left\{a_{j}: t^{j}=t_{i}\right\}$. Then $\bar{a}_{i}$ is the desired tuple. such that $\bar{a}_{i} \in \mathscr{A}_{t_{i}}$ and $\bar{a}_{i} \cap \bigcup_{t<t_{i}} \mathscr{A}_{i}=\emptyset$.

$\nabla_{\text {Subclaim 3.13.2.1 }}$

To save ink, we will denote the tuples $\bar{a}_{i}$ as $a_{i}$, and now consider the finite set of tuples $\left\{a_{0}, \ldots, a_{n}\right\}$.

We wish to refine this choice of witnesses $\left\{a_{0}, \ldots, a_{n}\right\}$ to minimize the $t_{n}$ and the number $n$. To this end, we devise an ordering on $\mathscr{P} \leqslant \omega\left(K_{i}\right)$ :

Definition 3.14. For $t_{i}, u_{i} \in K_{i}$, we say that $\left\{t_{i}: i \leqslant n\right\} \Subset\left\{u_{i}: i \leqslant m\right\}$ iff for all $i \leqslant n$ there is $j \in m$ such that $t_{i} \leq u_{j}$ and there is $u_{j}$ such that $u_{j} \not \leq t_{i}$ for every $i \leqslant n$.

We can minimize the choice of witnesses $\left\{a_{0}, \ldots, a_{n}\right\}$ easily if there are only finitely many candidates which may be smaller than our initial choice. We will assume otherwise, and, using Ramsey's Theorem, come to a contradiction. Thus, assume for a contradiction, that there are infinitely many choices of witnesses $\left\{a_{0}, \ldots, a_{n}\right\}=\left\{a_{0}^{0}, \ldots, a_{n_{0}}^{0}\right\},\left\{a_{0}^{1}, \ldots, a_{n_{1}}^{1}\right\}, \ldots,\left\{a_{0}^{j}, \ldots, a_{n_{j}}^{j}\right\}, \ldots$ from $\mathscr{P}<\omega(K)$ for which the associated $\left\{t_{0}, \ldots, t_{n}\right\}=\left\{t_{0}^{0}, \ldots, t_{n_{0}}^{0}\right\},\left\{t_{0}^{1}, \ldots, t_{n_{1}}^{1}\right\}, \ldots$, $\left\{t_{0}^{j}, \ldots, t_{n_{j}}^{j}\right\}, \ldots$, are $\Subset$ than our original choice. These are quasi-ordered by $\Subset$.

Subclaim 3.14.0.2. The collection

$$
\left\{t_{0}^{0}, \ldots, t_{n_{0}}^{0}\right\},\left\{t_{0}^{1}, \ldots, t_{n_{1}}^{1}\right\}, \ldots,\left\{t_{0}^{j}, \ldots, t_{n_{j}}^{j}\right\}, \ldots
$$

is a quasi-ordering with no $\Subset$-infinite descending sequences.

For notational simplicity, we will write $X_{j}=\left\{t_{i}^{j}: i<n_{j}\right\}$, and consider them with the ordering $\Subset$.

Assume for a contradiction that there is an infinite descending chain. We assume, without loss of generality, that this chain is enumerated so that $X_{j+1} \Subset$ $X_{j}$.

Let $u_{j} \in X_{j}$ be such that $u_{j} \not^{K} t_{k}^{j+1}$ for every $k<n_{j+1}$ (by definition of $\Subset$, there is at least one such $u_{j} \in X_{j}$ for every $j$ ).

Thus, for all $j<i<\omega, u_{j} \not^{K} u_{i}$. This is because if $i=j+1$, then this is simply the definition of $u_{j}$, and otherwise, we can find $k<n_{j+1}$ such that $u_{i} \leq^{K} t_{k}^{j+1}$. So, if $u_{j} \leq^{K} u_{i}$, then $u_{j} \leq^{K} t_{k}^{j+1}$, a contradiction with the definition of $u_{i}$. 
Since the $u_{j}$ are finite antichains in $J_{i}$, it is easy to see that $\bigcup\left\{u_{j}: j<\omega\right\}$ does not contain infinite decreasing $\leq^{J}$-chains. By the same argument, there are also no infinite increasing $\leq^{J}$-sequences.

By Ramsey's Theorem, there must thus be an infinite $\leq^{J}$-antichain. Thus, we can find $t_{i}^{0}, i<n_{0}$, and an infinite set $X \subseteq \omega$ such that $\left\{u_{j}: j \in X\right\}$ is an $\leq^{K}$-antichain, and $u_{j} \leq{ }^{K} t_{i}^{0}$ for all $j \in X$.

Let $T$ be the tree composed of $\eta \in J$, such that $\eta<\xi$ for some $\xi \in t_{i}^{0} \subset J$. We show that since such a tree has no maximal branches, the existence of an infinite $\leq{ }^{K}$-antichain is not possible.

Note that for all $j<i$ and $k$, there is $n$ such that $t_{k}^{i} \leq{ }^{K} t_{n}^{j}$.

Without loss of generality, we can assume that $u_{j}=\left\{u_{i}^{j}: i<m\right\}$. To ensure this, we may need to make $X$ smaller so that $\left|u_{j}\right| \leqslant n_{0}$, for all $i \in X$.

By applying the Ramsey Theorem $m$ times, we can assume the one of the following for all $i<m$ :

(1) for all $j<k, u_{i}^{k}<^{K} u_{i}^{k}$;

(2) for all $j<k, u_{i}^{j} \perp^{K} u_{i}^{k}$;

(3) for all $j<k, u_{i}^{j} \geq^{K} u_{i}^{k}$.

Clearly case 1 is not possible. Furthermore, it is not possible for case 3 holds for all $i<m$. Thus, let $i<m$ be such that 2 holds. Then $\left\{u_{i}^{j}: j \in X\right\}$ is an infinite $\leq^{J}$-antichain in $T$, a contradiction.

$\nabla_{\text {Subclaim 3.14.0.2 }}$

Assume now that our choice of $\left\{a_{0}, \ldots, a_{n}\right\}$ and $\left\{t_{0}, \ldots, t_{n}\right\}$ is minimal in $<^{K}$. There is $C \subset \bigcup_{t<t_{n}} \mathscr{A}_{t},|C|=\lambda_{r}\left(\mathbf{m}_{\mathbf{l}}\right)$ such that

$$
\operatorname{tp}\left(a_{n} / C\right) \models \operatorname{tp}\left(a_{n} / \bigcup_{t<t_{n}} \mathscr{A}_{t}\right) .
$$

Let $B=C \cup\left\{a_{0}, \ldots, a_{n-1}\right\}$.

On the one hand, let $H=f_{\left(t_{n}, t_{0}, \ldots, t_{n-1}\right)}^{B}$ be as in Definition 3.9. That is, $H$ is an automorphism of $\mathbf{m}$ such that $H \uparrow_{\left(B \cap \mathscr{A}_{t_{n}}\right)}=i d_{B \cap \mathscr{A}_{t_{n}}}$ and for $i<n$,

$$
H\left(B \cap \mathscr{A}_{t_{i}}\right) \subseteq \mathscr{A}_{\operatorname{MinSetIn}\left(t_{n}, t_{i}\right)}
$$

Then, $H\left(a_{i}\right) \in \mathscr{A}_{\operatorname{MinSetIn}\left(t_{n}, t_{i}\right)}$. Since $\operatorname{MinSetIn}\left(t_{n}, t_{i}\right)<t_{n}, H\left(a_{i}\right) \in \bigcup_{t^{\prime}<t_{n}} \mathscr{A}_{t^{\prime}}$. Since $H \uparrow_{C}=i d$, we have

$$
\operatorname{tp}\left(a_{0}, \ldots, a_{n-1} / C\right)=\operatorname{tp}\left(H\left(a_{0}\right), \ldots, H\left(a_{n-1}\right) / C\right)
$$

and

$$
\operatorname{tp}\left(a_{n} / C\right) \models \operatorname{tp}\left(a_{n} / C \cup\left\{H\left(a_{0}\right), \ldots, H\left(a_{n-1}\right)\right\}\right),
$$

SO

$$
\operatorname{tp}\left(a_{n} / C\right) \models \operatorname{tp}\left(a_{n} / C \cup\left\{a_{0}, \ldots, a_{n-1}\right\}\right) .
$$

On the other hand, consider $G^{*}$. Since $\left\{a_{0}, \ldots, a_{n}\right\}$ is a minimal witness that $G^{*}$ is not elementary, the function $G^{*}\left\lceil C \cup\left\{a_{0}, \ldots, a_{n-1}\right\}\right.$ must be elementary.

Let $G^{+}$be an automorphism of $\boldsymbol{m}$ such that $G^{+} \circ G^{*}\left\lceil_{C \cup\left\{a_{0}, \ldots, a_{n-1}\right\}}=i d\right.$. 
Since $G^{*} \uparrow_{\mathscr{A}_{t_{n}}}=G_{t_{n}}$ and $C \subseteq \mathscr{A}_{t_{n}}, G^{*} \uparrow_{C \cup a_{n}}$ is elementary. Thus

$$
\operatorname{tp}\left(G^{+}\left(G^{*}\left(a_{n}\right)\right) / C\right)=\operatorname{tp}\left(a_{n} / C\right) .
$$

However,

$$
\operatorname{tp}\left(G^{+}\left(G^{*}\left(a_{n}\right)\right), a_{0}, \ldots, a_{n-1} / C\right)=\operatorname{tp}\left(G^{*}\left(a_{n}\right), G^{*}\left(a_{0}\right), \ldots, G^{*}\left(a_{n-1}\right) / G^{*}(C)\right),
$$

thus

$$
\operatorname{tp}\left(G^{*}\left(a_{n}\right), G^{*}\left(a_{0}\right), \ldots, G^{*}\left(a_{n-1}\right) / \emptyset\right) \models \operatorname{tp}\left(a_{n}, a_{0}, \ldots, a_{n-1} / \emptyset\right) .
$$

This means that

a contradiction.

$$
\operatorname{tp}\left(a_{n} / C\right) \forall=\operatorname{tp}\left(a_{n} / C \cup a_{0}, \ldots, a_{n-1}\right),
$$

Corollary 3.15. Let $\mathscr{A}^{J_{0}}$ and $\mathscr{A}^{J_{1}}$ be models constructed as above for trees $J_{0}$ and $J_{1}$. Assume that in a cardinal-preserving extension of the universe, $S\left(J_{0}\right)$ is not stationary. Then $\mathscr{A}^{J_{0}} \cong \mathscr{A}^{J_{1}}$.

Proof. Lemmas 7.15 and 7.31 of [HHR04] demonstrate that in the extension, $J_{0} \cong J_{1}$. We can then apply the previous theorem 3.13 .

3.5. Constructibility with respect to $0^{\#}$. We now have all the necessary ingredients to prove Theorem 3.1.

Proof. The result is a direct result of Theorem 2.4 and Corollaries 3.12 and 3.15.

\section{REFERENCES}

[FHR03] Sy David Friedman, Tapani Hyttinen, and Mika Rautila. Classification theory and $0^{\#}$. Journal of Symbolic Logic, 68(2):580-588, 2003.

[Fri03] Sy D. Friedman. Cardinal-preserving extensions. Journal of Symbolic Logic, 68(4):1163-1170, 2003.

[GL02] Rami Grossberg and Olivier Lessmann. Shelah's stability spectrum and homogeneity spectrum in finite diagrams. Archive for Mathematical Logic, 41(1):1-31, 2002.

[GS86] Rami Grossberg and Saharon Shelah. On the number of nonisomorphic models of an infinitary theory which has the infinitary order property. Part A. The Journal of Symbolic Logic, 51(2):302-322, 1986.

[HHR04] Taneli Huuskonen, Tapani Hyttinen, and Mika Rautila. On potential isomorphism and non-structure. Archive for Mathematical Logic, 43(1):85-120, 2004.

[HS98] Tapani Hyttinen and Saharon Shelah. On the number of elementary submodels of an unsuperstable homogeneous structure. Math Logic Quarterly, 44:354-358, 1998.

[HS00] Tapani Hyttinen and Saharon Shelah. Strong splitting in stable homogeneous models. Annals of Pure and Applied Logic, 103(1-3):201-228, 2000.

[HS01] Tapani Hyttinen and Saharon Shelah. Main gap for locally saturated elementary submodels of a homogeneous structure. Journal of Symbolic Logic, 66(3):1286-1302, 2001.

[HT91] Tapani Hyttinen and Heikki Tuuri. Constructing strongly equivalent nonisomorphic models for unstable theories. Annals of Pure and Applied Logic, 52(3):203-248, 1991. 
[Hyt97a] Tapani Hyttinen. On nonstructure of elementary submodels of an unsuperstable homogeneous structure. Mathematical Logic Quarterly, 43(1):134-142, 1997.

[Hyt97b] Tapani Hyttinen. A Short Introduction to Classification Theory, volume 2 of Graduate Texts in Mathematics. Department of Mathematics, University of Helsinki, Helsinki, 1997.

[Jec03] Thomas Jech. Set theory. Springer Monographs in Mathematics. Springer-Verlag, Berlin, 2003. The third millennium edition, revised and expanded.

[She70] Saharon Shelah. Finite diagrams stable in power. Annals of Mathematical Logic, 2:69118, 1970.

[She87] Saharon Shelah. Existence of many $L_{\infty, \lambda}$-equivalent, nonisomorphic models of $T$ of power $\lambda$. Annals of Pure and Applied Logic, 34:291-310, 1987.

[She90] Saharon Shelah. Classification theory and the number of non-isomorphic models, volume 92 of Studies in Logic and the Foundations of Mathematics. North-Holland, Amsterdam, 2nd, revised edition, 1990.

Sy-DAVID Friedman

Kurt Gödel Research Center for Mathematical Logic

UNIVERSITY OF VIENNA

A-1090 Vienna, Austria

E-mail address: sdf@logic.univie.ac.at

TAPANi HyTtinen

Department of Mathematics and Statistics

UNIVERSITY OF HELSINKI

FI-00014 Helsinki, Finland

E-mail address: tapani.hyttinen@helsinki.fi

Agatha C. Walczak-Typke

Department of Mathematics and Statistics

UNIVERSITY OF HELSINKI

FI-00014 Helsinki, Finland

E-mail address: agatha.walczak-typke@helsinki.fi 